\title{
PANCREATIC CYST FLUID GLUCOSE: RAPID, INEXPENSIVE, AND ACCURATE DIAGNOSIS OF MUCINOUS PANCREATIC CYSTS
}

\author{
Rosalie A. Carr MD ${ }^{1,6}$, Michele T Yip-Schneider PhD ${ }^{1,3,4,6}$, Rachel E. Simpson MD ${ }^{1,6}$, Scott \\ Dolejs MD ${ }^{1}$, Justine G. Schneider ${ }^{1}$, Huangbing Wu BS ${ }^{1,6}$, Eugene P. Ceppa MD ${ }^{1,4,6}$, Walter \\ Park $\mathrm{MD}^{5}$, C. Max Schmidt MD, PhD, MBA ${ }^{1-4,6}$ \\ Departments of ${ }^{1}$ Surgery and ${ }^{2}$ Biochemistry/Molecular Biology, Indiana University School of \\ Medicine, ${ }^{3}$ Walther Oncology Center, ${ }^{4}$ Indiana University Cancer Center, ${ }^{5}$ Department of \\ Medicine, Stanford University, Stanford, CA, ${ }^{6}$ Indiana University Health Pancreatic Cyst and \\ Cancer Early Detection Center, Indianapolis, IN
}

Presented at the Central Surgical Association, Chicago, IL, July 2017

\section{Correspondence Address:}

C. Max Schmidt, MD, PhD, MBA, FACS

Vice Chairman of Surgery, Academic Affairs

Chief of Surgery, IUH University Hospital

Director, IUH Pancreatic Cyst and Cancer Early Detection Center

Professor, Surgery, Biochemistry \& Molecular Biology

Indiana University School of Medicine

545 Barnhill Drive EH 129

Indianapolis, IN 46202

maxschmi@iupui.edu | www.pancyst.org

Office 317.948.8358 | Cell 317.372.9011 | Fax 317.274.0241

OR

Michele T. Yip-Schneider, PhD

Associate Research Professor

Department of Surgery, Indiana University School of Medicine

980 W. Walnut St., Building R3, Rm. 541C

Indianapolis, IN 46202

Phone: (317) 278-4739 FAX: (317) 274-0396

myipschn@iupui.edu

This is the author's manuscript of the article published in final edited form as:

Carr, R. A., Yip-Schneider, M. T., Simpson, R. E., Dolejs, S., Schneider, J. G., Wu, H., ... Schmidt, C. M. (2018). Pancreatic cyst fluid glucose: rapid, inexpensive, and accurate diagnosis of mucinous pancreatic cysts. Surgery, 163(3), 600-605.

https://doi.org/10.1016/j.surg.2017.09.051 


\section{Abstract}

Background: The most widely accepted biochemical test for preoperative differentiation of mucinous from benign, non-mucinous pancreatic cysts is cyst fluid carcinoembryonic antigen (CEA). However, the diagnostic accuracy of CEA ranges from 70-86\%. Based on previous work, we hypothesize that pancreatic cyst fluid glucose may be an attractive alternative to CEA. Methods: Pancreatic cyst fluid was collected during endoscopic or operative intervention. Diagnoses were pathologically confirmed. Glucose and CEA were measured using a patient glucometer and automated analyzer/ELISA. Sensitivity, specificity, accuracy, and ROC analyses were performed.

Results: Cyst fluid samples from 153 patients were evaluated (mucinous: 25 mucinous cystic neoplasm, 77 intraductal papillary mucinous neoplasm, 4 ductal adenocarcinoma; non-mucinous: 21 serous cystic neoplasm, 9 cystic neuroendocrine tumor, 14 pseudocyst, 3 solid pseudopapillary neoplasm). Median cyst fluid glucose was lower in mucinous versus nonmucinous cysts (19 vs 96mg/dL; $\mathrm{p}<0.0001$ ). With a threshold of $\leq 50 \mathrm{mg} / \mathrm{dL}$, cyst fluid glucose was $92 \%$ sensitive, $87 \%$ specific, and $90 \%$ accurate in diagnosing mucinous pancreatic cysts. In comparison, cyst fluid CEA with a threshold of $>192 \mathrm{ng} / \mathrm{ml}$ was $58 \%$ sensitive, $96 \%$ specific, and 69\% accurate. AUC for glucose and CEA were similar at 0.91 and 0.92 .

Conclusion: Cyst fluid glucose has significant advantages over CEA and should be considered for use as a routine diagnostic test for pancreatic mucinous cysts. 


\section{$\underline{\text { Introduction }}$}

Pancreatic cancer will be diagnosed in 53,670 Americans and will take the lives of 43,090 in $2017^{1}$. Current available treatment strategies offer little chance for cure and a limited extension of life. In light of the low long-term survival rates following pancreatic cancer diagnosis, optimal clinical management should include prevention strategies. A unique opportunity for prevention of pancreatic cancer exists in specific high-risk populations such as patients with precancerous pancreatic cysts. While as many as 2-3\% of American adults are found to have pancreatic cysts on routine cross-sectional imaging, not all cysts have malignant potential and undergo malignant transformation ${ }^{2,3}$. Patients known to have cysts with a high risk for malignant transformation will optimally be managed surgically. Those with lower risk cysts may be followed with more or less intensive surveillance programs depending on risk stratification. Avoidance of unnecessary, highly morbid surgery balanced with prevention of pancreatic cancer hinges on accurate preoperative diagnosis and malignant risk stratification.

Diagnostic tools for pancreatic cysts are limited by variable accuracy and reliability. While cross-sectional imaging can detect the vast majority of pancreatic cysts, its accuracy in differentiating cyst types is lacking ${ }^{4}$. Differentiation of cyst types is key because this in part will determine their malignant potential. Mucinous pancreatic cystic lesions include intraductal papillary mucinous neoplasms (IPMN) and mucinous cystic neoplasms (MCN), both of which can undergo malignant progression ${ }^{5}$. Conversely, non-mucinous cysts include serous cystic neoplasms (SCN) and pseudocysts with virtually no propensity for malignancy and cystic pancreatic neuroendocrine tumors (PNET) and solid pseudopapillary neoplasms (SPN) which are rare and almost always identified accurately on cytology. To aid in risk stratification, endoscopic ultrasound with fine needle aspiration is often performed in order to obtain cyst fluid for 
biomarker, cytologic, and genetic analysis ${ }^{5}$. Cyst fluid carcinoembryonic antigen (CEA) is the standard biomarker currently used to differentiate mucinous from non-mucinous pancreatic cysts

${ }^{6}$. However, CEA is not perfect. A recent multi-institutional retrospective study found CEA sensitivity and specificity of only $61 \%$ and $77 \%$ at the accepted $192 \mathrm{ng} / \mathrm{mL}$ threshold for detection of mucinous cystic lesions ${ }^{7}$. Previous meta-analysis reported similar findings of $63 \%$ and $88 \%$ sensitivity and specificity of CEA ${ }^{8}$. Furthermore, CEA measurement requires specific laboratory capabilities that are costly and relatively time consuming.

We hypothesize that an alternate cyst fluid biomarker may offer improved diagnostic accuracy and efficiency over the standard CEA test for determination of mucinous versus nonmucinous cysts. Two previous studies from a single institution reported the potential of pancreatic cyst fluid glucose for the diagnosis of mucinous cysts ${ }^{9,10}$. The studies included 45 and 65 patient samples and found sensitivities and specificities ranging from 81-95\% and 57$78 \%$ for detection of mucinous pancreatic cysts using thresholds of $<66$ and $50 \mathrm{mg} / \mathrm{dL}$, respectively. We aim to independently validate these findings with a larger patient cohort and to compare the diagnostic utility of cyst fluid glucose and CEA.

\section{$\underline{\text { Methods }}$}

Pancreatic cyst fluid samples were collected prospectively at the time of endoscopic ultrasound guided fine needle aspiration $\underline{(\mathrm{n}=41)}$ or pancreatic resection $\underline{(\mathrm{n}=112)}$ at Indiana University Health University Hospital between June 2003 and June 2016. All patients provided informed consent in accordance with the Indiana University Institutional Review Board. After procurement, pancreatic cyst fluid aliquots were placed immediately on ice and then stored at $80^{\circ}$ C. Pancreatic cyst diagnosis was confirmed on surgical pathology by a University Hospital Staff pathologist and then reconfirmed by a pancreatic pathologist. Demographic and clinical 
data were prospectively collected as patient samples were gathered. Additional or missing variables were obtained from retrospective review of electronic medical records.

\section{Glucose and CEA Analysis}

Pancreatic cyst fluid ( $2 \mu \mathrm{l})$ was thawed on ice and assayed within 1 hour. Glucose was analyzed using a standard patient glucometer, OneTouch VerioIQ Blood Glucose Monitoring System. The OneTouch glucometer measures glucose levels between $20 \mathrm{mg} / \mathrm{dL}$ and $600 \mathrm{mg} / \mathrm{dL}$

${ }^{11}$. No pancreatic cyst fluid sample had a glucose reading of $>600 \mathrm{mg} / \mathrm{dL}$. All samples with glucose readings $<20 \mathrm{mg} / \mathrm{dL}$ were recorded and analyzed as $19 \mathrm{mg} / \mathrm{dL}$. A subset of patient samples had adequate fluid for concomitant CEA analysis. CEA was determined by Beckman Coulter DxI 800 analyzer or in cases of low fluid volume, by ELISA (Sigma-Aldrich, St. Louis, MO). CEA values obtained by ELISA were converted to the Beckman automated analyzer scale using linear regression.

\section{Statistical Analysis}

Descriptive statistics including mean, median, standard deviation, interquartile range, and frequencies were calculated as appropriate. Demographic and clinic-pathologic data were compared between patients with mucinous and non-mucinous pancreatic cysts using MannWhitney U-test for continuous data and Chi Square for categorical data. Pearson correlation coefficient was calculated to determine the association of glucose with other variables. P-values of $<0.05$ were considered statistically significant. The diagnostic utility of glucose as a biomarker for mucinous cystic lesions was ascertained using sensitivity/specificity calculations and receiver operator characteristic (ROC) analyses. Analyses were repeated for cyst fluid CEA and compared to glucose analyses.

\section{$\underline{\text { Results }}$}


A total of 153 pancreatic cyst fluid samples were collected and analyzed for study inclusion. Of these, 106 were pathologically confirmed as mucinous (25 mucinous cystic neoplasm, 77 intraductal papillary mucinous neoplasm, 4 ductal adenocarcinoma) and 47 as nonmucinous cysts (21 serous cystic neoplasm, 9 cystic neuroendocrine tumor, 14 pseudocyst, and 3 solid pseudopapillary neoplasm). Although patient gender did not differ between those with mucinous and non-mucinous cysts ( $31.7 \%$ vs $27.7 \%$ male; $\mathrm{p}=0.7)$, median age (interquartile range, IQR) was significantly younger in the non-mucinous cyst group at 65 (55-73) years versus 58 (42-68) years (Table 1). Frequency of diabetes mellitus (23.8\% vs. 27.7\%), insulin use (9.4\% vs. $8.5 \%$ ), median serum hemoglobin a1c (Ha1c) (5.9 vs. 5.8), and median cyst size (2.8 cm vs. $3.6 \mathrm{~cm}$ ) were also not different between mucinous versus non-mucinous cyst groups (Table 1). None of the above patient demographic or clinical variables correlated with pancreatic cyst fluid glucose or CEA.

Median pancreatic cyst fluid glucose level [IQR] measured using a standard patient glucometer was significantly lower in mucinous cysts than in non-mucinous cysts (19 [19-29] vs. 96 [66-114] mg/dL; $\mathrm{p}<0.0001$ ) (Figure 1). Blood glucose levels on the day of the collection procedure did not correlate with cyst fluid glucose levels (data not shown). Figure 2 displays the scatter plot dividing mucinous and non-mucinous cyst categories into cyst types comprising each category. Median and IQR values are shown under the scatter plot. All median values for mucinous cyst types (MCN, IPMN, and PDAC) fall below the previously published cutoff value (50 mg/dL) for detection of mucinous cysts ${ }^{9}$. With a threshold of $<50 \mathrm{mg} / \mathrm{dL}$, cyst fluid glucose was $92 \%$ sensitive, $87 \%$ specific, and $90 \%$ accurate in diagnosing mucinous pancreatic cysts. Receiver operator curve analysis was performed revealing an area under the curve (AUC) of 0.91 (95\% CI: 0.85-0.96) (Figure 3). Pancreatic cyst fluid glucose was unable to differentiate 
invasive disease from non-invasive disease, or invasive IPMN from non-invasive IPMN. Additionally, no association was found between cyst fluid glucose levels and indications for surgery such as IPMN dysplasia grade or main duct involvement (data not shown).

Of the 153 patients analyzed for glucose, 120 had sufficient pancreatic cyst fluid volume available for CEA measurement. Median cyst fluid CEA was significantly higher in mucinous cysts than in non-mucinous cysts (471.7 [63.7-1638.5] vs. 1.0 [0.1-14.6] ng/mL, $\mathrm{p}<0.001)$ (Figure 4). When using the standard threshold value of $>192 \mathrm{ng} / \mathrm{mL}$ for identifying mucinous cystic lesions, sensitivity, specificity, and accuracy were 58\%, 96\%, and 69\%. On ROC analysis, AUC was 0.92, not significantly different from the glucose AUC of 0.91 ( $\mathrm{p}=0.8)$. Combining glucose and CEA for differentiating mucinous from non-mucinous pancreatic cysts had a sensitivity of $95 \%$, specificity of $85 \%$, accuracy of $93 \%$, and AUC of 0.95 . The AUC for all three tests (glucose alone, CEA alone, and glucose/CEA combination) were similar with the only statistical difference being the combination test performed slightly better than glucose alone $(\mathrm{p}=0.03)$.

\section{$\underline{\text { Discussion }}$}

International consensus guidelines for the management of mucinous pancreatic cysts (IPMN and MCN) were published in 2006 and updated in 2012 to aide clinicians in the practice of evidence-based cyst management ${ }^{5,12}$. Diagnostic recommendations encourage initial use of clinical and imaging characteristics, specifically CT and MRCP which may identify classic findings of a particular type of cyst, e.g., IPMN- main duct dilation, main pancreatic duct connection, and multiplicity. On the contrary, the solitary cyst is more difficult to characterize (mucinous vs non-mucinous) on imaging. If high risk features (i.e. cyst size $>3 \mathrm{~cm}$, the presence of mural modularity, or main duct dilation) are detected by imaging, then endoscopic ultrasound 
guided fine needle aspiration can be used to obtain cyst fluid for chemical and cytologic analysis. The international consensus guidelines include several specific cyst fluid analyses: CEA, amylase, cytology, KRAS, and GNAS. Although these are the most widely recognized cyst fluid biomarkers for diagnosis of mucinous lesions, all are flawed. Cytologic analyses are severely limited by lack of cyst fluid cellularity ${ }^{5}$. Pancreatic cyst fluid KRAS mutation is an extremely specific test (96\%) for mucinous lesions, but lacks sensitivity (45\%) ${ }^{13}$. Combined with cyst fluid GNAS mutation, sensitivity and specificity reportedly increase to $65 \%$ and $100 \%$ respectively ${ }^{14}$.

In addition to those cyst fluid analyses recommended by the international consensus guidelines, other potential tests to identify mucinous pancreatic cysts have been reported. Mucin staining has been used for mucinous cyst differentiation with sensitivity and specificity of $80 \%$ and $40 \%{ }^{15}$. A study of cyst fluid viscosity demonstrated elevated measurements in mucinous cysts although IPMN were not included ${ }^{16}$. The string sign is performed by placing a drop of fluid between two fingers and slowly spreading the fingers to stretch the fluid. Formation of a string greater than $1 \mathrm{~cm}$ lasting one second was $85 \%$ specific and $95 \%$ sensitive for the diagnosis of mucinous cysts in a study by Bick and colleagues ${ }^{17}$. The presence of various mucins within pancreatic cyst fluid detected mucinous lesions with comparable diagnostic accuracy ${ }^{18,19}$.

The most widely used pancreatic cyst fluid biomarker is CEA ${ }^{6}$. At the standard threshold value of $>192 \mathrm{ng} / \mathrm{mL}$, CEA can differentiate mucinous from non-mucinous type cysts with an accuracy of $77 \%$ according to a large multi-institutional validation study of 1,861 patients ${ }^{7}$. Smaller studies report accuracy ranging from $70-86 \%$, sensitivity of $61-89 \%$, and specificity of $63-77 \%{ }^{20-23}$. However, CEA alone lacks sufficient accuracy for routine identification of mucinous cysts ${ }^{13,24,25}$. In the current study, CEA $>192 \mathrm{ng} / \mathrm{mL}$ had an accuracy of $69 \%$. Although specificity was high at $96 \%$, sensitivity was inadmissibly low at $58 \%$. Not 
only is CEA alone insufficiently accurate as a biomarker, the optimal cutoff for a positive test is controversial and may vary based on the specific laboratory or automated analyzer performing the analysis. For the present dataset, with an alternate threshold of $\geq 26 \mathrm{ng} / \mathrm{mL}$ chosen to maximize diagnostic performance, CEA sensitivity, specificity, and accuracy for mucinous cyst detection was $86 \%$, $85 \%$, and $86 \%$ respectively. Others have evaluated the diagnostic

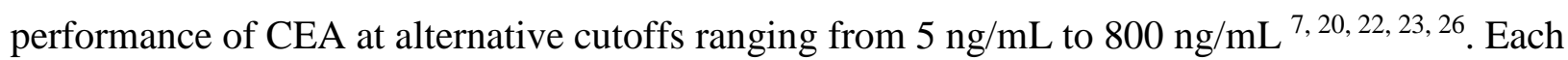
reported comparable or slightly improved diagnostic accuracy of CEA at the alternative thresholds, thus illustrating the need for careful interpretation of CEA values in the clinical setting.

Here we present glucose as an attractive pancreatic cyst fluid biomarker for the detection of mucinous cysts. Our findings confirm previously reported high diagnostic performance of glucose $^{9,10}$. At $<50 \mathrm{mg} / \mathrm{dL}$, glucose accurately diagnosed mucinous pancreatic cysts in $90 \%$ of cases. CEA accuracy was less at $69 \%$ and $86 \%$ depending on the threshold values selected as discussed above. In addition to the improved accuracy of glucose over CEA, the reproducibility of glucose is advantageous. As previously mentioned, optimal cutoff values for CEA vary widely perhaps due in part to the various automated analyzers/methods used to measure CEA at the institution(s). In contrast, glucose can be measured using a simple, commercially available patient glucometer. Using the same type/brand of glucometer at medical centers/clinics would result in higher reproducibility. Our results were analyzed using the $50 \mathrm{mg} / \mathrm{dL}$ glucose threshold established by Stanford University; pending future validation by other groups, this cut-off may be applied universally leading to more uniform interpretation of test results.

Additionally, the glucometer is simple to use, designed for use by patients who have no biomedical training. Conversely, CEA measurement takes place in a laboratory by trained 
laboratory personnel using highly specialized materials and equipment. The cost of a standard glucometer ranges from ten to seventy dollars; single-use test strips cost from ten cents to two dollars depending on brand. The glucometer and test strips used in this study cost thirty dollars and $\$ 1.60$ each, respectively ${ }^{11}$. Divided among the 153 tested samples in the current study, per patient cost was $\$ 1.80$. Real cost in the clinical setting would be lower still, due to many more uses per glucometer and discounted materials associated with purchase of greater quantities. For CEA, although actual cost of materials for each measurement is low ( \$2.65), the Beckman Coulter DxI 800 analyzer used at Indiana University has a high initial cost of $\$ 150,000$. The expensive equipment, trained staff, equipment servicing, and facilities result in high cost to the patient. Accordingly, patients at Indiana University are charged \$142 to have cyst fluid CEA measured in the clinical laboratory. Other advantages to measuring cyst fluid glucose include the rapidity (seconds) and very low volume required $(2 \mu \mathrm{l}$ vs. $>200 \mu \mathrm{l}$ for CEA). The latter is a very important consideration given that large volumes of cyst fluid are often difficult to obtain, thus precluding cyst fluid CEA analysis.

In current literature, the most accurate tests for detection of mucinous pancreatic cysts are combination tests ${ }^{15-17,22,26-29}$. The majority of these combination tests include CEA along with one to four additional biomarkers. Reported combination tests range from simple, involving only two individual tests, to extremely complicated, employing use of sequential test interpretation models. Endoscopic ultrasound, cytology, and CEA combined (positive test defined as any one positive single test) differentiates mucinous from non-mucinous pancreatic cysts with sensitivity, specificity, and accuracy of $91 \%, 75 \%$, and $86 \%$ according to Oppong and colleagues ${ }^{26}$. More elaborate is the sequential test interpretation model designed by Bick and colleagues in which cytology, mucin staining, CEA, and string sign analyses are performed chronologically ${ }^{17}$. If any 
component test is positive, the entire test is considered positive, and the remainder of component tests are not performed. Only if all four component tests are negative is the entire test negative. Using this methodology, mucinous cysts were identified with 88\% sensitivity, 92\% specificity, and 89\% accuracy. Park and colleagues utilized a novel panel of protein biomarkers (afamin, lithostathine-1-alpha, and polymeric immunoglobulin receptor) to achieve an area under the curve of 0.933. In the present study, if cyst fluid glucose and CEA tests were combined to maximize diagnostic potential, accuracy improved to 93\% with an AUC of 0.95. Combining glucose with including additional biomarkers, clinical factors, and imaging characteristics may further enhance its performance as a biomarker of mucinous cysts.

This study was limited by several factors. All patients in this series underwent surgical pancreatic resection. As many patients with pancreatic cysts never receive operative care or may even go undiagnosed, data collected from surgical patients may not be generalizable to all cyst patients. The surgical indication might also contribute to cyst fluid glucose levels (i.e. symptoms, size, growth). Cyst size was analyzed in previous studies and the current study and did not correlate with cyst fluid glucose concentration. Additional prospective studies in which fluid analysis is performed preoperatively are needed to simulate anticipated clinical use. Finally, these results should be further validated in a large, prospective multi-institution study.

\section{Conclusions}

Pancreatic cyst fluid glucose differentiates mucinous from non-mucinous cysts with similar accuracy to the current "gold-standard”, CEA. However, glucose testing has several distinct advantages in that it is simple, rapid, inexpensive, and requires minimal cyst fluid. Thus, cyst fluid glucose should routinely be tested to aid in the diagnosis of mucinous pancreatic cysts. 
Combining CEA and glucose improves diagnostic accuracy and may further approach perfection if evaluated together with additional biomarkers, clinical factors, and imaging characteristics.

\section{Financial Disclosures}

No authors have financial disclosures or conflicts of interest to report. 


\section{$\underline{\text { References }}$}

1. Howlader N, Noone A, Krapcho M, Miller D, Bishop K, Kosary C, et al. SEER Cancer Statistics Review, 1975-2014, National Cancer Institute. Bethesda, MD. 2016.

2. Soroida Y, Sato M, Hikita H, Hagiwara S, Sato M, Gotoh H, et al. Pancreatic cysts in general population on ultrasonography: Prevalence and development of risk score. Journal of gastroenterology. 2016;51:1133-40.

3. Chang YR, Park JK, Jang JY, Kwon W, Yoon JH, Kim SW. Incidental pancreatic cystic neoplasms in an asymptomatic healthy population of 21,745 individuals: Large-scale, single-center cohort study. Medicine. 2016;95:e5535.

4. Palmucci S, Cappello G, Trombatore C, Tilocca C, Todaro R, Mauro LA, et al. Cystic pancreatic neoplasms: diagnosis and management emphasizing their imaging features. European review for medical and pharmacological sciences. 2014;18:1259-68.

5. Tanaka M, Fernandez-del Castillo C, Adsay V, Chari S, Falconi M, Jang JY, et al. International consensus guidelines 2012 for the management of IPMN and MCN of the pancreas. Pancreatology : official journal of the International Association of Pancreatology (IAP) [et al]. 2012;12:183-97.

6. Brugge WR, Lewandrowski K, Lee-Lewandrowski E, Centeno BA, Szydlo T, Regan S, et al. Diagnosis of pancreatic cystic neoplasms: a report of the cooperative pancreatic cyst study. Gastroenterology. 2004;126:1330-6.

7. Gaddam S, Ge PS, Keach JW, Mullady D, Fukami N, Edmundowicz SA, et al. Suboptimal accuracy of carcinoembryonic antigen in differentiation of mucinous and 
nonmucinous pancreatic cysts: results of a large multicenter study. Gastrointestinal endoscopy. 2015;82:1060-9.

8. Thornton GD, McPhail MJ, Nayagam S, Hewitt MJ, Vlavianos P, Monahan KJ. Endoscopic ultrasound guided fine needle aspiration for the diagnosis of pancreatic cystic neoplasms: a meta-analysis. Pancreatology : official journal of the International Association of Pancreatology (IAP) [et al]. 2013;13:48-57.

9. Zikos T, Pham K, Bowen R, Chen AM, Banerjee S, Friedland S, et al. Cyst Fluid Glucose is Rapidly Feasible and Accurate in Diagnosing Mucinous Pancreatic Cysts. The American journal of gastroenterology. 2015;110:909-14.

10. Park WG, Wu M, Bowen R, Zheng M, Fitch WL, Pai RK, et al. Metabolomic-derived novel cyst fluid biomarkers for pancreatic cysts: glucose and kynurenine. Gastrointestinal endoscopy. 2013;78:295-302.e2.

11. OneTouch Verio IQ Blood Glucose Monitoring System Owner's Booklet. Switzerland: LifeScan Europe; 2011.

12. Tanaka M, Chari S, Adsay V, Fernandez-del Castillo C, Falconi M, Shimizu M, et al. International consensus guidelines for management of intraductal papillary mucinous neoplasms and mucinous cystic neoplasms of the pancreas. Pancreatology : official journal of the International Association of Pancreatology (IAP) [et al]. 2006;6:17-32.

13. Khalid A, Zahid M, Finkelstein SD, LeBlanc JK, Kaushik N, Ahmad N, et al. Pancreatic cyst fluid DNA analysis in evaluating pancreatic cysts: a report of the PANDA study. Gastrointestinal endoscopy. 2009;69:1095-102.

14. Singhi AD, Nikiforova MN, Fasanella KE, McGrath KM, Pai RK, Ohori NP, et al. Preoperative GNAS and KRAS testing in the diagnosis of pancreatic mucinous cysts. 
Clinical cancer research : an official journal of the American Association for Cancer Research. 2014;20:4381-9.

15. Morris-Stiff G, Lentz G, Chalikonda S, Johnson M, Biscotti C, Stevens T, et al. Pancreatic cyst aspiration analysis for cystic neoplasms: mucin or carcinoembryonic antigen--which is better? Surgery. 2010;148:638-44; discussion 44-5.

16. Linder JD, Geenen JE, Catalano MF. Cyst fluid analysis obtained by EUS-guided FNA in the evaluation of discrete cystic neoplasms of the pancreas: a prospective single-center experience. Gastrointestinal endoscopy. 2006;64:697-702.

17. Bick BL, Enders FT, Levy MJ, Zhang L, Henry MR, Abu Dayyeh BK, et al. The string sign for diagnosis of mucinous pancreatic cysts. Endoscopy. 2015;47:626-31.

18. Maker AV, Katabi N, Gonen M, DeMatteo RP, D'Angelica MI, Fong Y, et al. Pancreatic cyst fluid and serum mucin levels predict dysplasia in intraductal papillary mucinous neoplasms of the pancreas. Annals of surgical oncology. 2011;18:199-206.

19. Jabbar KS, Verbeke C, Hyltander AG, Sjovall H, Hansson GC, Sadik R. Proteomic mucin profiling for the identification of cystic precursors of pancreatic cancer. Journal of the National Cancer Institute. 2014;106:djt439.

20. van der Waaij LA, van Dullemen HM, Porte RJ. Cyst fluid analysis in the differential diagnosis of pancreatic cystic lesions: a pooled analysis. Gastrointestinal endoscopy. 2005;62:383-9.

21. Oh SH, Lee JK, Lee KT, Lee KH, Woo YS, Noh DH. The Combination of Cyst Fluid Carcinoembryonic Antigen, Cytology and Viscosity Increases the Diagnostic Accuracy of Mucinous Pancreatic Cysts. Gut and liver. 2016. 
22. Nagashio Y, Hijioka S, Mizuno N, Hara K, Imaoka H, Bhatia V, et al. Combination of cyst fluid CEA and CA 125 is an accurate diagnostic tool for differentiating mucinous cystic neoplasms from intraductal papillary mucinous neoplasms. Pancreatology : official journal of the International Association of Pancreatology (IAP) [et al]. 2014;14:503-9.

23. Cizginer S, Turner BG, Bilge AR, Karaca C, Pitman MB, Brugge WR. Cyst fluid carcinoembryonic antigen is an accurate diagnostic marker of pancreatic mucinous cysts. Pancreas. 2011;40:1024-8.

24. Nagula S, Kennedy T, Schattner MA, Brennan MF, Gerdes H, Markowitz AJ, et al. Evaluation of cyst fluid CEA analysis in the diagnosis of mucinous cysts of the pancreas. Journal of gastrointestinal surgery : official journal of the Society for Surgery of the Alimentary Tract. 2010;14:1997-2003.

25. Brugge WR. Cyst fluid: moving beyond the carcinoembryonic antigen. Gastrointestinal endoscopy. 2015;82:1070-1.

26. Oppong KW, Dawwas MF, Charnley RM, Wadehra V, Elamin K, White S, et al. EUS and EUS-FNA diagnosis of suspected pancreatic cystic neoplasms: Is the sum of the parts greater than the CEA? Pancreatology : official journal of the International Association of Pancreatology (IAP) [et al]. 2015;15:531-7.

27. Kadayifci A, Atar M, Wang JL, Forcione DG, Casey BW, Pitman MB, et al. Value of adding GNAS testing to pancreatic cyst fluid KRAS and carcinoembryonic antigen analysis for the diagnosis of intraductal papillary mucinous neoplasms. Digestive endoscopy : official journal of the Japan Gastroenterological Endoscopy Society. 2016. 
28. Al-Haddad M, DeWitt J, Sherman S, Schmidt CM, LeBlanc JK, McHenry L, et al. Performance characteristics of molecular (DNA) analysis for the diagnosis of mucinous pancreatic cysts. Gastrointestinal endoscopy. 2014;79:79-87.

29. Park J, Yun HS, Lee KH, Lee KT, Lee JK, Lee SY. Discovery and Validation of Biomarkers That Distinguish Mucinous and Nonmucinous Pancreatic Cysts. Cancer research. 2015;75:3227-35. 
Table 1: Patient Demographic/Clinical Data for Those with Mucinous vs. Non-mucinous Pancreatic Cysts

\begin{tabular}{|l|c|c|c|c|}
\hline & n & Mucinous & Non-mucinous & p-value \\
\hline Gender (\% male) & 153 & $31.7 \%$ & $27.7 \%$ & 0.7 \\
\hline Age [Median (IQR)] & 153 & $65.0(55.0-73.0)$ & $58.0(42.0-68.0)$ & 0.006 \\
\hline DM (\% with DM) & 153 & $23.8 \%$ & $27.7 \%$ & 0.7 \\
\hline Insulin use & 153 & $9.4 \%$ & $8.5 \%$ & 0.5 \\
\hline Ha1c & 74 & $5.9(5.7-6.6)$ & $5.8(5.3-6.0)$ & 0.06 \\
\hline Cyst size (cm) & 151 & $2.8(2.1-4.3)$ & $3.6(2.5-5.3)$ & 0.08 \\
\hline
\end{tabular}

DM: Diabetes Mellitus

Ha1c: Hemoglobin a1c 


\section{FIGURE LEGENDS}

\section{Figure 1: Pancreatic Cyst Fluid Glucose in Mucinous vs. Non-mucinous Pancreatic Cysts} Cyst fluid glucose (y-axis) is compared between mucinous and non-mucinous cysts on the $\mathrm{x}$ axis. The thick horizontal bars represent median values. Bordering median bars are thin, interquartile range bars. The horizontal dashed line identifies the cutoff value of $50 \mathrm{mg} / \mathrm{dL}$ for detecting mucinous pancreatic cysts.

\section{Figure 2: Pancreatic Cyst Fluid Glucose for All Categories of Cyst Types}

Pancreatic cyst fluid glucose (y-axis) is plotted for each cyst according to pancreatic cyst type (xaxis). Mucinous cysts, shown on the left side of the graph, include MCN, IPMN, and PDAC. Non-mucinous cysts, shown on the right side of the graph, include SCN, NET, pseudocyst, and SPN. The thick horizontal bars represent median values. Bordering median bars are thin, interquartile range bars. The horizontal dashed line identifies the cutoff value of 50mg/dL for detecting mucinous pancreatic cysts. All mucinous cyst median values fall below this cutoff. Median and IQR values for each cyst type are shown below the scatter plot.

\section{Figure 3: Pancreatic Cyst Fluid Glucose Receiver Operator Characteristic (ROC) Curve}

\section{Analysis}

The AUC for cyst fluid glucose is 0.91 .

\section{Figure 4: Pancreatic Cyst Fluid CEA}

Cyst fluid CEA (y-axis) is compared between mucinous and non-mucinous cysts on the x-axis. CEA values are plotted on a log linear scale. The thick horizontal bars represent median values. 
Bordering median bars are thin, interquartile range bars. The horizontal dashed line identifies the standard cutoff value of $192 \mathrm{ng} / \mathrm{mL}$ for detecting mucinous pancreatic cysts. 


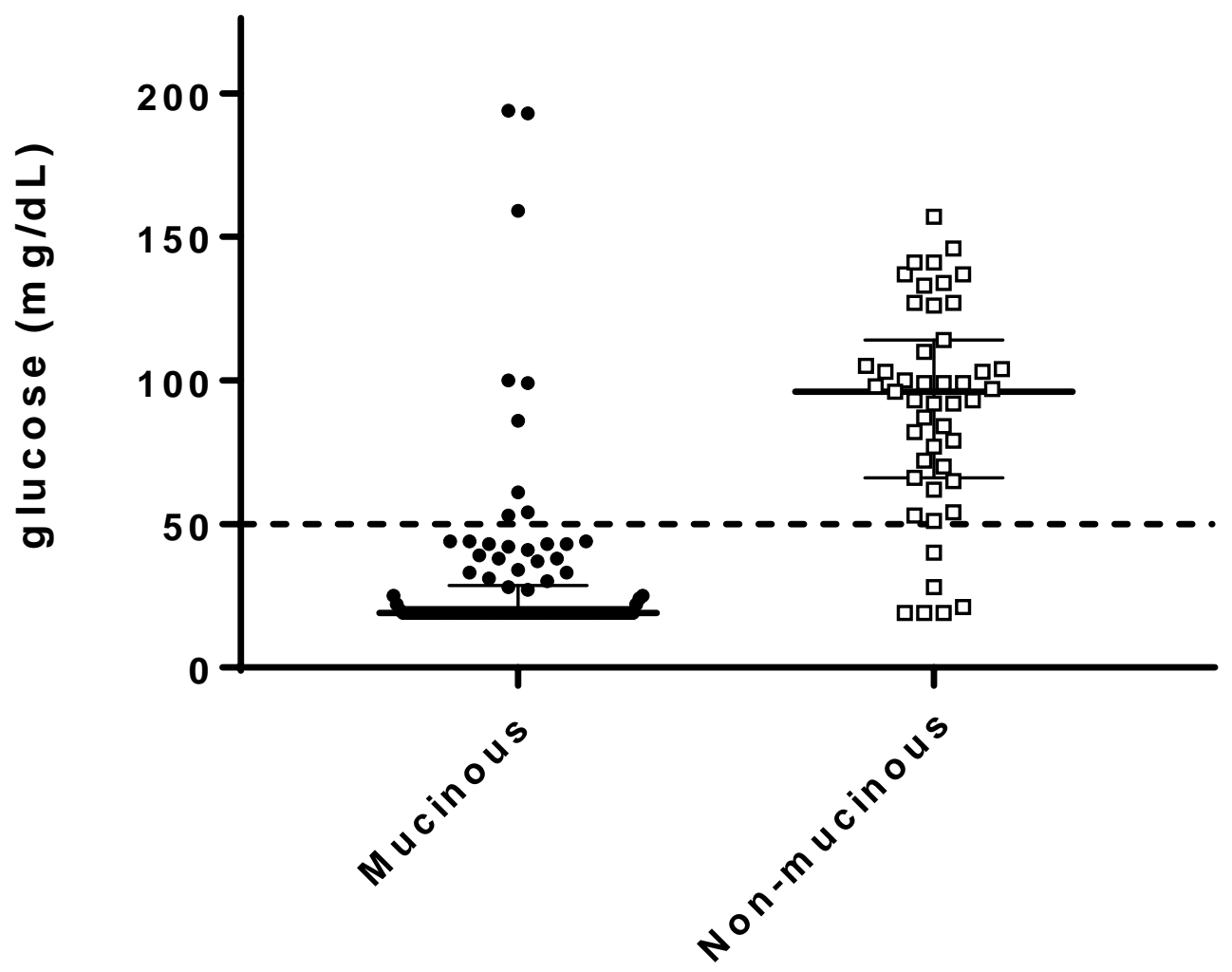




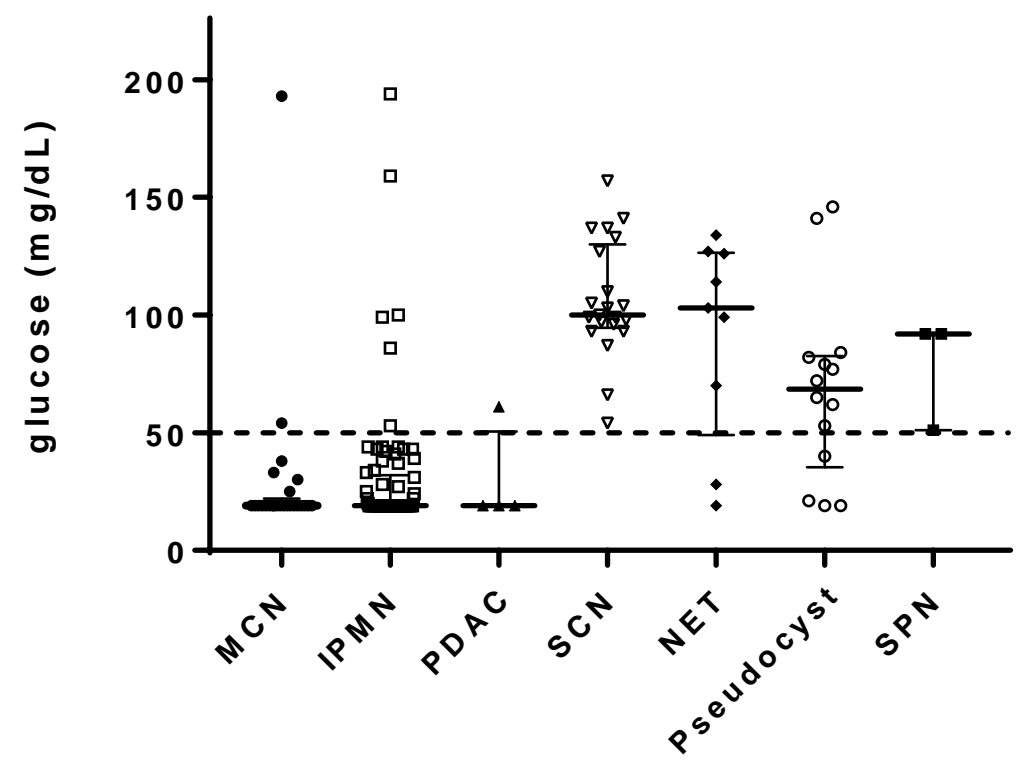

\begin{tabular}{|l|c|c|}
\hline \multicolumn{1}{|c|}{ Pancreatic Cyst Type } & $\begin{array}{c}\text { Median glucose } \\
\text { (mg/dL) }\end{array}$ & $\begin{array}{c}\text { IQR } \\
\text { (Interquartile range) }\end{array}$ \\
\hline MCN (mucinous cystic neoplasm) & 19 & $19-22$ \\
\hline IPMN (intraductal papillary mucinous neoplasm) & 19 & $19-32$ \\
\hline PDAC (pancreatic ductal adenocarcinoma) & 19 & $19-51$ \\
\hline SCN (serous cystic neoplasm) & 100 & $95-130$ \\
\hline NET (neuroendocrine tumor) & 103 & $49-127$ \\
\hline Pseudocyst & 69 & $35-83$ \\
\hline SPN (solid pseudopapillary neoplasm) & 92 & $51-92$ \\
\hline
\end{tabular}




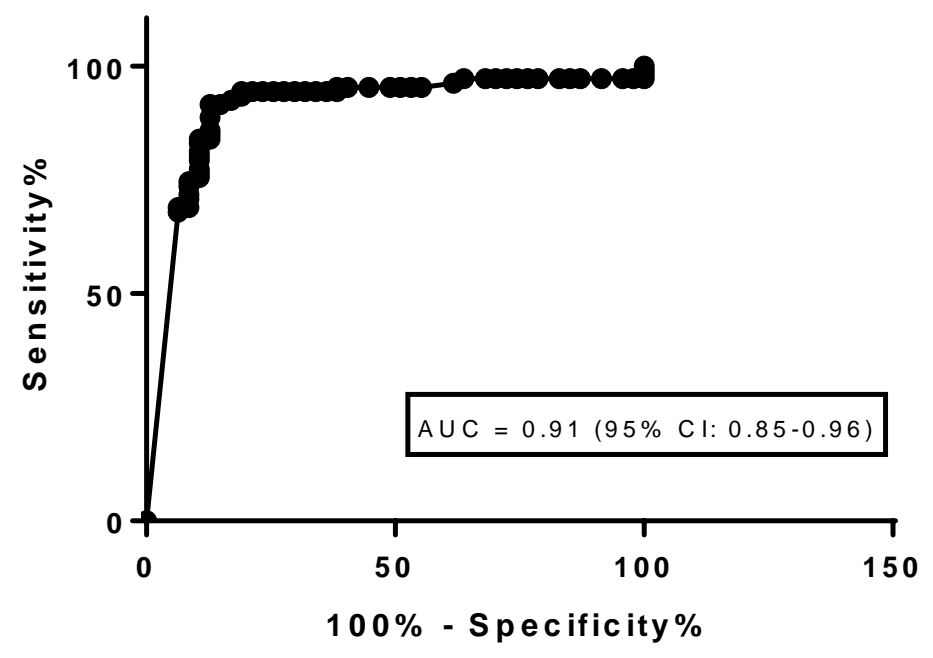




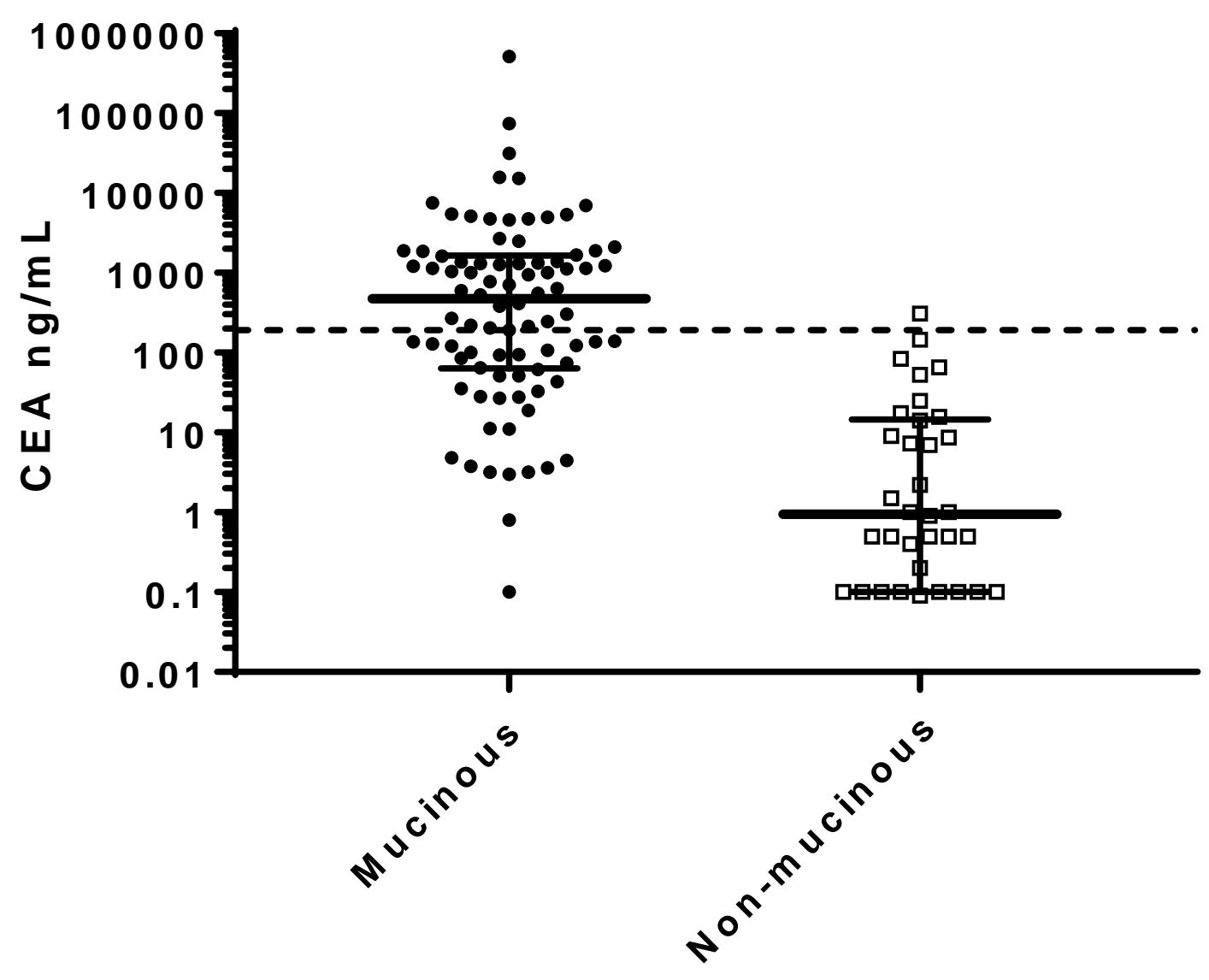

